\title{
Safety and Efficiency of Mini-Sternotomy versus Standard Sternotomy in Open Heart Surgery for Aortic Valve Replacement
}

\author{
Essam Saad Abdelwahed, Ahmed Mohamed Amin Bakry, Ahmed Elwan Mohamed and Ahmed Alhammali \\ Amir Abdulsalam \\ Department of Cardiothoracic Surgery, Faculty of Medicine - Zagazig University, Egypt. \\ *Corresponding Author: Ahmed Alhammali Amir Adulsalam, Mobile: 01552266507, Email: dr.aalhammali@gmail.com
}

\begin{abstract}
Background: For decades, the traditional median sternotomy has been the access of choice in aortic valve surgery; however, numerous researchers have worked to develop less invasive procedures, and the partial upper ministernotomy is the most prevalent minimally invasive procedure. Objective: To compare outcomes, in patients who undergo mini-sternotomy, and patients who undergo standard sternotomy, in aortic valve replacement operations. Patients and Methods: This randomized controlled clinical trial study included fifty patients who were admitted to the Zagazig University Hospital, Cardiothoracic Surgery Department in the period between January 2021 to August 2021, Patients were split into two groups, each with 25 patients: Group "A" had aortic valve surgery by ministernotomy, while Group "B" got aortic valve surgery via traditional surgery. Aortic valve surgery was performed via a conventional median sternotomy in Group "B." An ECG was performed to see whether there was any ischemia present, as well as laboratory tests and a chest $\mathrm{x}$-ray. Results: In the present research, there was a significant difference in postoperative outcomes between the mini-sternotomy and complete sternotomy groups in terms of mechanical ventilation and hospital stay. Inotropic support and reopening were not substantially different across groups; however, infection and blood transfusion were strongly linked with the complete group, and neither group had any mortality. Conclusions: Minimally invasive aortic valve replacement, is a safe and effective procedure and is performed with comparable morbidity and mortality to conventional aortic valve replacement (AVR). Minimally invasive aortic valve replacement (MIAVR) results in improved ventilator function, fewer wound infections, shorter hospital stays, and a higher percentage of patients released home early.
\end{abstract}

Keywords: Mini Sternotomy, Full Sternotomy, Aortic Valve Replacement.

\section{INTRODUCTION}

The majority of patients with severe aortic valve stenosis are elderly individuals with several comorbidities, resulting in a greater surgical risk profile and associated morbidity and mortality risks ${ }^{(\mathbf{1}, 2)}$. Harken $\boldsymbol{e t}$ al. published the first report on aortic valve replacement in 1960, and it has since become the second most common cardiac surgical operation, beyond coronary artery bypass surgery ${ }^{(3)}$. In an attempt to avoid the dangers of a full sternotomy, minimally invasive aortic valve replacement became popular in the 1990s and was found to be related with less postoperative discomfort, blood loss, pulmonary issues, wound complications, and a shorter hospital stay $(4,5)$.

\section{PATIENTS AND METHODS}

Fifty patients with aortic valve disease who needed aortic valve surgery were chosen nonrandomly. The research was carried out in the Cardiothoracic Department of Zagazig University Hospital.

Twenty-five patients had standard sternotomy for aortic valve surgery, whereas the other twenty-five patients underwent mini-sternotomy $\mathrm{J}$ shape to the third right intercostal space with aortic and single venous cannulation.

\section{Ethical considerations:}

An approval of the study was obtained from Zagazig University Academic and Ethical Committee. Every patient signed an informed written consent for acceptance of the operation and sharing in this study.
This work has been carried out in accordance with The Code of Ethics of the World Medical Association (Declaration of Helsinki) for studies involving humans (IRB\#6628/30-12-2020).

Inclusion criteria: Age > 16. Patients who are candidates for isolated aortic valve replacement, either aortic valve stenosis or aortic valve regurgitation, or both.

Exclusion criteria: Patients suffering from severe coronary artery disease. Renal failure sufferers. Patients experiencing cardiogenic shock or cardiopulmonary resuscitation prior to surgery. Patients who have had a redo or combination valve surgery. Patients with abnormalities of the chest or vertebral walls. When aortic root dilatation is required in patients with a small aortic annulus. Infectious endocarditis.

\section{Technique:}

For all patients, the intraoperative anesthetic approach was the same: a 20-gauge non-dominant radial artery cannula was placed utilizing local anesthesia. The first blood sample was taken from the arterial line for preoperative baseline activated clotting time (ACT) analysis, and the second was taken for preoperative baseline arterial blood gas (ABG) analysis. Two grey peripheral venous cannulas were used for monitoring, followed by Fentanyl $5-10 \mathrm{mic} / \mathrm{kg}$ and Rocuronium (Esmeron) 0.6-1.2 $\mathrm{mg} / \mathrm{kg}$ as a loading dosage and $0.1-0.2 \mathrm{mg} / \mathrm{kg}$ as a maintenance dose for endotracheal intubation and a propofol hypnotic dose of $1-2.5 \mathrm{mg} / \mathrm{kg}$ augmented hypnotic dosage. The trachea 
was intubated orally with an adequately sized endotracheal tube after complete muscular relaxation. Inhalational isoflurane, MAC (0.5-1.0 percent), was used to maintain anesthesia in all patients, following induction.

\section{Cardiopulmonary bypass (CPB):}

Membrane oxygenators were employed in this experiment. During CPB, the hematocrit was maintained at approximately $28 \%$. Systemic cooling to 28 degrees Celsius was used to preserve the myocardium. In case of aortic valve stenosis, antegrade warm blood cardioplegia was fed into the ascending aorta via a cardioplegia cannula with pressure, or directly into the coronary ostia in case of aortic valve regurge, within 1-3 minutes, an induced cardiac standstill was typically obtained. Cardioplegia was administered every 30-40 minutes at a dosage of 10 $\mathrm{ml} / \mathrm{Kg}$.

\section{Group "A" (MIAVS); J shaped mini-sternotomy:}

On the operating table, the patient was positioned in a supine posture with his or her arms by his or her side. Under the shoulders, a sandbag was placed.

The patient was then wrapped in the traditional manner, with the chest skin and groin regions exposed. a 5-8 cm midline skin incision from one finger below the sternal notch to the level of the 3rd intercostal space, then dissection with an electrocautery blade until the sternal periosteum is reached In the upper manubrium, a conventional sternal saw was engaged, directed caudally, and ultimately turned a side to the right into the $3^{\text {rd }}$ intercostal space. Electrocautery was used to reduce bleeding from the sternal periosteum. In instances of severe marrow bleeding, bone wax was utilized. The cross arm of a tiny sternal retractor was positioned in the lower end of the incision and the retractor was gently opened. The sternum was only opened as far as it needed to be for sufficient exposure. After dissecting the thymus gland, the pericardium was accessed. Suturing the pericardium to the margins of the incision with strong silk sutures typically provides sufficient exposure. Aortic cannulation was done with a 20 or 22 straight aortic cannula, followed by single venous cannulation and aortic root cannulation for cardioplegia delivery and de-airing.

After initiating cardiopulmonary bypass, and after dealing with the aortic valve lesion by either repair or replacement, before coming off bypass, placing chest tube was done by inserting a retrosternal tube, and pleural tubes if needed. Our department policy recommends that retrosternal tubes should not be removed before $48 \mathrm{hrs}$ from the time of procedure. Weaning from the cardiopulmonary bypass, decannulation and hemostasis was obtained, pacing wires was then inserted. The sternum was then approximated using five heavy stainless-steel wires, which was passed through the sternum. The twisted wires were then carefully turned down into the sternum so that they do not protrude externally. The linea alba was approximated with heavy absorbable suture as was the pectoralis fascia. The subcutaneous tissue was then closed by continuous absorbable $2 / 0$ sutures, followed by the skin, which was closed by $3 / 0$ subcuticular suture.

\section{Group "B" (Full Sternotomy):}

In a supine posture, the patient was put. Palpation was used to locate the sternal notch and the distal point of the xiphoid process, the incision was made in the sternal notch and prolonged $2 \mathrm{~cm}$ beyond the distal tip of the xiphoid process, with electrocautery utilized to extend it to the sternal periosteum. The incision was then widened in the midline using electrocautery between the insertion sites of the pectoralis major muscle. The linea alba was divided above the suprasternal ligament at the xiphoid, and a plane behind the sternum was created by blunt finger dissection. The sternum was then divided in a cephalad to caudal direction by hooking the nose-plate of the saw underneath the suprasternal ligament, and by lifting the sternum upwards and asking the anesthesiologist to deflate the lungs, it was safer to avoid injury of the underlying pleura. Bleeding from sternal periosteum was then controlled by electrocautery. Bone wax was used in cases with excessive marrow bleeding. A sternal retractor with broad blades was placed and opened slowly, with the cross arm of the retractor positioned in the upper end of the incision.

\section{Postoperative follow up and evaluation:}

All patients were evaluated thoroughly during their intensive care unit stay and during their hospital stay. Intensive care unit evaluation: weaning of mechanical ventilation Continuous positive airway pressure (CPAP) and pressure support (10-15 $\mathrm{cm} \mathrm{H}_{2} \mathrm{O}$ ) modes were used progressively.

Ventilatory support was gradually decreased at a rate of 1-2 cm $\mathrm{H}_{2} \mathrm{O}$ CPAP decrements. Postoperative blood loss during the ICU stays and till the chest tubes were removed was calculated in both groups. Patients were evaluated after surgery by chest X-ray postero-anterior view, duration of mechanical ventilation, total ICU stay, total hospital stay, transfusion requirements.

\section{Statistic analysis}

The data collected during the history, basic clinical examination, laboratory tests, and outcome assessments were coded, entered, and analyzed using Microsoft Excel software. The data were analyzed using the Statistical Package for the Social Sciences (SPSS version 20.0). Chi square test was used for difference and association of qualitative variables that were presented as number and percentage. To assess differences between quantitative, which were presented as mean \pm standard deviation (SD), independent-t test was employed. The $\mathrm{P}$ value for significant findings was set at 0.05 , while the $\mathrm{P}$ value for highly significant results was set at 0.001 .

\section{RESULTS}

Table 1 revealed that there was no significant difference between groups in terms of age, BMI or sex distribution. 
Table (1): Demographic data distribution between studied groups

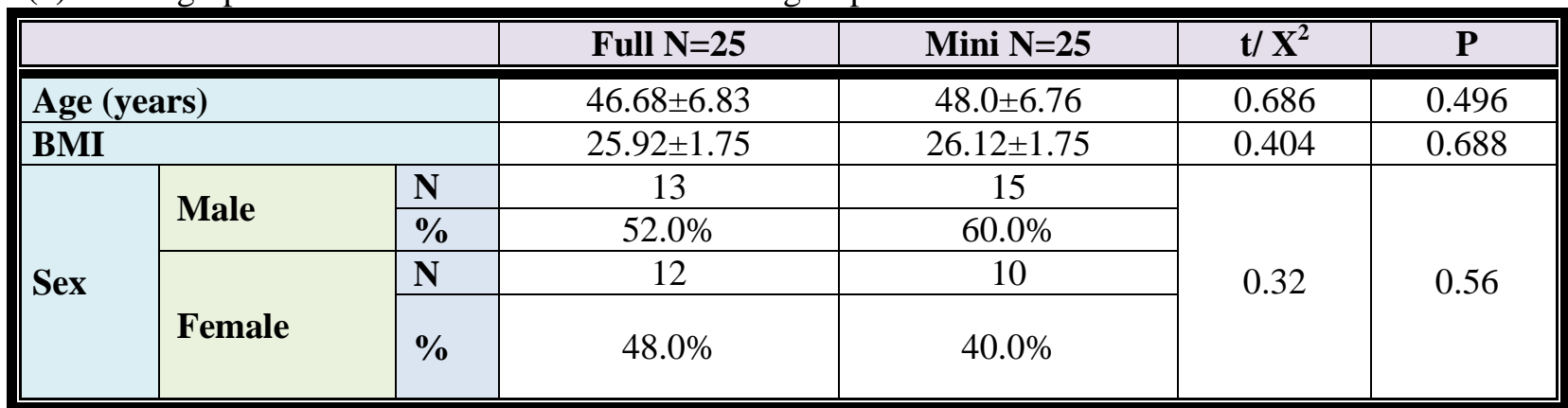

There was no significant difference or relationship between groups in terms of comorbidities, with hypertension being the most common comorbidity across both groups (Table 2).

Table (2): Comorbidities distribution between studied groups

\begin{tabular}{|c|c|c|c|c|c|c|}
\hline \multirow{4}{*}{ Diabetes mellitus } & VT & $\mathbf{N}$ & 19 & 17 & \multirow{4}{*}{0.4} & \multirow{4}{*}{0.53} \\
\hline & $-V E$ & $\%$ & $76.0 \%$ & $68.0 \%$ & & \\
\hline & \multirow{2}{*}{$+\mathrm{VE}$} & $\mathbf{N}$ & 6 & 8 & & \\
\hline & & $\%$ & $24.0 \%$ & $32.0 \%$ & & \\
\hline \multirow{4}{*}{ Hypertension } & \multirow{2}{*}{$-\mathrm{VE}$} & $\mathbf{N}$ & 14 & 17 & \multirow{4}{*}{0.76} & \multirow{4}{*}{0.38} \\
\hline & & $\%$ & $56.0 \%$ & $68.0 \%$ & & \\
\hline & \multirow{2}{*}{$+\mathrm{VE}$} & $\mathbf{N}$ & 11 & 8 & & \\
\hline & & $\%$ & $44.0 \%$ & $32.0 \%$ & & \\
\hline \multirow{4}{*}{ Dyslipidemia } & VF & $\mathbf{N}$ & 21 & 19 & \multirow{4}{*}{0.50} & \multirow{4}{*}{0.48} \\
\hline & $-\sqrt{2}$ & $\%$ & $84.0 \%$ & $76.0 \%$ & & \\
\hline & \multirow{2}{*}{$+\mathrm{VE}$} & $\mathbf{N}$ & 4 & 6 & & \\
\hline & & $\%$ & $16.0 \%$ & $24.0 \%$ & & \\
\hline \multirow{2}{*}{\multicolumn{2}{|c|}{ Total }} & $\mathbf{N}$ & 25 & 25 & & \\
\hline & & $\%$ & $100.0 \%$ & $100.0 \%$ & & \\
\hline
\end{tabular}
mini group.

Table 3 shows that aortic cross clamp time, total bypass time and operative time were significantly longer in

Table (3): Comparison of aortic cross clamp time, total bypass time and operative time between studied groups

\begin{tabular}{|l|c|c|c|c|}
\hline & Full & Mini & t & P \\
\hline \hline Aortic cross clamp time & $53.24 \pm 7.21$ & $67.92 \pm 10.33$ & 5.822 & $<0.001 * *$ \\
\hline Total bypass time & $71.76 \pm 10.41$ & $83.68 \pm 11.34$ & 3.869 & $<0.001^{* *}$ \\
\hline Operative time & $185.0 \pm 16.99$ & $251.60 \pm 16.22$ & 14.174 & $<0.001 * *$ \\
\hline
\end{tabular}

**: Highly significant

Table 4 shows that there was significant difference regarding MV duration as it was significantly shorter in mini group, and hospital stay was significantly longer among full cases, and total drain was significantly higher in full group.

Table (4): MV duration, ICU and hospital stay distribution between studied groups

\begin{tabular}{|l|c|c|c|c|}
\hline & Full & Mini & t & P \\
\hline \hline Duration of MV/ Hours & $9.78 \pm 3.14$ & $7.32 \pm 1.78$ & 2.116 & $0.041^{*}$ \\
\hline ICU stay /Hours & $46.44 \pm 8.22$ & $42.32 \pm 5.25$ & 2.112 & $0.04^{*}$ \\
\hline Hospital stay / days & $6.76 \pm 1.20$ & $5.44 \pm 1.50$ & 3.433 & $0.001^{* *}$ \\
\hline Total drain/ml & $506.85 \pm 90.52$ & $304.63 \pm 80.69$ & 7.589 & $0.00^{* *}$ \\
\hline
\end{tabular}

MV: Mechanical ventilation, ICU: Intensive care unit, *: Significant, **: Highly significant

Table 5 shows that there was no significant difference between groups as regard inotropic support and reopening, but infection and blood transfusion were significantly associated with full group and we reported no mortality at both groups. 
Table (5): Outcome distribution at pre and post between studied groups

\begin{tabular}{|c|c|c|c|c|c|c|}
\hline & & & \multicolumn{2}{|c|}{ Group } & \multirow{2}{*}{$\mathbf{X}^{2}$} & \multirow{2}{*}{$\mathbf{P}$} \\
\hline & & & Full & Mini & & \\
\hline \multirow{4}{*}{ Inotropic support } & \multirow{2}{*}{ No } & $\mathbf{N}$ & 22 & 23 & \multirow{4}{*}{0.22} & \multirow{4}{*}{0.64} \\
\hline & & $\%$ & $88.0 \%$ & $92.0 \%$ & & \\
\hline & \multirow{2}{*}{ Yes } & $\mathbf{N}$ & 3 & 2 & & \\
\hline & & $\%$ & $12.0 \%$ & $8.0 \%$ & & \\
\hline \multirow{4}{*}{ Reopening } & \multirow{2}{*}{ No } & $\mathbf{N}$ & 24 & 25 & \multirow{4}{*}{1.02} & \multirow{4}{*}{0.31} \\
\hline & & $\%$ & $96.0 \%$ & $100.0 \%$ & & \\
\hline & \multirow{2}{*}{ Yes } & $\mathbf{N}$ & 1 & 0 & & \\
\hline & & $\%$ & $4.0 \%$ & $0.0 \%$ & & \\
\hline \multirow{4}{*}{ Infection } & \multirow{2}{*}{ No } & $\mathbf{N}$ & 20 & 25 & \multirow{4}{*}{5.56} & \multirow{4}{*}{$0.02 *$} \\
\hline & & $\%$ & $80.0 \%$ & $100.0 \%$ & & \\
\hline & \multirow{2}{*}{ Yes } & $\mathbf{N}$ & 5 & 0 & & \\
\hline & & $\%$ & $20.0 \%$ & $0.0 \%$ & & \\
\hline \multirow{8}{*}{ Transfusion requirement } & \multirow{2}{*}{0.00} & $\mathbf{N}$ & 3 & 5 & \multirow{8}{*}{12.01} & \multirow{8}{*}{$0.007 *$} \\
\hline & & $\%$ & $12.0 \%$ & $20.0 \%$ & & \\
\hline & \multirow{2}{*}{1.00} & $\mathbf{N}$ & 8 & 17 & & \\
\hline & & $\%$ & $32.0 \%$ & $68.0 \%$ & & \\
\hline & \multirow{2}{*}{2.00} & $\mathbf{N}$ & 8 & 3 & & \\
\hline & & $\%$ & $32.0 \%$ & $12.0 \%$ & & \\
\hline & \multirow{2}{*}{3.00} & $\mathbf{N}$ & 6 & 0 & & \\
\hline & & $\%$ & $24.0 \%$ & $0.0 \%$ & & \\
\hline \multirow{2}{*}{\multicolumn{2}{|c|}{ Total }} & $\mathbf{N}$ & 25 & 25 & & \\
\hline & & $\%$ & $100.0 \%$ & $100.0 \%$ & & \\
\hline
\end{tabular}

*: Significant

\section{DISCUSSION}

There was no significant difference or correlation between groups in terms of comorbidities in the current study, and hypertension was the most common comorbidity across both groups. The percent of diabetic patients in another study in the upper ministernotomy group was 48 percent, whereas the standard full sternotomy group was 40 percent, with no statistical significance, and the percent of hypertensive patients in the upper mini-sternotomy group was 60 percent, whereas the standard full sternotomy group was 68 percent, with no statistical significance ${ }^{(6)}$.

In the current research, the upper mini-sternotomy group had significantly longer aortic cross clamp duration, total bypass time, and surgical time than the standard sternotomy group. Another study found that the range of aortic cross clamp time (minutes) in the upper mini-sternotomy group was $42-116 \mathrm{~min}$, with a mean of $81.44 \pm 16.92 \mathrm{~min}$, whereas the range in the standard full sternotomy group was 45-68 minutes (min), with a mean of $60.04 \pm 5.82 \mathrm{~min}$, with statistical significance. The upper mini-sternotomy group had a total bypass time (min) range of 64-140 min, with a mean of $107.48 \pm 20.06 \mathrm{~min}$, whereas the standard full sternotomy group had a range of 70-86 min, with a mean of $75.76 \pm 4.06 \mathrm{~min}$, with statistical significance. The upper mini-sternotomy group had a total operative time range of $175-360$ minutes, with a mean of $308.48 \pm 65.51$ minutes, whereas the standard full sternotomy group had a total operative time range of 178-258 minutes, with a mean of $198.88 \pm 18.85$ minutes, with statistical significance ${ }^{(\mathbf{6})}$.

Our research found that the mini-sternotomy (MS) approach in AVR surgery has a narrow and unusual operative field, which results in a significantly longer operative time when compared to the full sternotomy (FS) approach in AVR due to a more cautious procedure, long preparation and cannulation time, long cardio-pulmonary bypass (CPB) time due to prolonged de-airing time, and time spent to insert substernal drains, and pacemaker wire. On the other hand upper mini-sternotomy, has a number of intraoperative advantages to full sternotomy, including a shorter time for hemostasis and fewer blood transfusions. In the present research, there was a significant difference in postoperative outcomes between the mini-sternotomy and complete sternotomy groups in terms of mechanical ventilation and hospital stay. Our department's standard is to keep the chest drains in place for at least 48 hours following surgery, thus there was no substantial difference in ICU stay between the two groups. In another study the length of time spent in the intensive care unit (ICU) was related to the severity of the illness. Stay in both groups was similar $(2.8 \pm 3.4$ days versus $2.3 \pm 1.4$ days in the Mini and Full sternotomy groups, respectively; $\mathrm{P}=$ 0.391). Patients in the mini group showing rapid recovery and had shorter hospital stays (7.6 2 days vs 
9.3 \pm 4.8 days; $\mathrm{P}=0.022$ ) than those in the complete sternotomy group. The fact that patients in the mini group spent less time in the hospital than those in the complete sternotomy group explained this agreement. The small group's quicker intrahospital recovery was aided by maintained chest wall integrity, less sternal wound problems, and easier postoperative mobility ${ }^{(7)}$.

MIAVR has been shown to have a quicker recovery time, shorter hospital stays, better aesthetic outcomes, and needs less rehabilitation resources as compared to traditional surgery ${ }^{(8)}$.

There was no important change between groups in terms of inotropic support or reopening in the present research, however infection and transfusion were substantially linked with the full sternotomy group, and neither group had any mortality. Another study found that the outcome of patients who had the upper mini-sternotomy method did not decrease the procedure's quality, and this technique is safe and effective for AVR ${ }^{(9)}$. There was a substantial variation in transfusion needs across the groups examined ${ }^{(\mathbf{1 0})}$.

In terms of hospital morbidity and mortality, limited access aortic valve surgery through an upper "J" sternotomy was just as safe as the conventional technique ${ }^{(\mathbf{1 1 1})}$.

The benefits of $\mathbf{J}$ shaped upper mini-sternotomy over traditional sternotomy included reduced surgical stress, bleeding, wound infections, and discomfort, as well as quicker patient recovery and favorable longterm results, especially in elderly, as compared to conventional sternotomy. Patients can move early and effectively because of the increased thoracic cage stability ${ }^{(12)}$.

MIAVR is a safe and effective treatment with a morbidity and mortality rate that is similar to traditional AVR ${ }^{(13)}$. MIAVR improves ventilator performance, decreases wound infection, lowers hospitalization time, and increases the percentage of patients who are sent home early.

\section{CONCLUSION}

We may conclude that minimally invasive AVR is a safe and successful surgery with few perioperative and short-term problems. Because it has numerous technical benefits, excellent clinical results, and is cost efficient, we think that mini-sternotomy is a viable option for standard sternotomy aortic valve surgery.
Financial support and sponsorship: Nil.

\section{Conflict of interest: Nil.}

\section{REFERENCES}

1. Iung B, Baron G, Tornos P et al. (2007): Valvular heart disease in the community: a European experience. Curr Probl Cardiol., 32: 609-661.

2. Mohr F, Holzhey D, Möllmann H et al. (2014): The German Aortic Valve Registry: 1-year results from 13,680 patients with aortic valve disease. Eur J Cardiothorac Surg., 46: 808-816.

3. Harken D, Taylor W, Lefemine A et al. (1962): Aortic valve replacement with a caged ball valve. Am J Cardiol., 9:292-29

4. Cosgrove M, Sabik F (1996): Minimally invasive approach for aortic valve operations. Ann Thorac Surg., 62: 596-597.

5. Rosengart K, Feldman T, Borger A et al. (2008): Percutaneous and minimally invasive valve procedures: a scientific statement from the American Heart Association Council on cardiovascular surgery and anesthesia, council on clinical cardiology, functional genomics and translational biology interdisciplinary working group, and quality of care and outcomes research interdisciplinary working group. Circulation, 117:1750-67.

6. Sarawy E, Kisho M, El Shemy A (2020): Comparative study between standard aortic valve replacement and ministernotomy aortic valve replacement. Journal of Medicine in Scientific Research, 3(3): 219-24.

7. Vukovic P, Milojevic P, Stojanovic I et al. (2019): The role of ministernotomy in aortic valve surgery-A prospective randomized study. J Card Surg., 34: 435-439.

8. Ferreira R, Silva R, Marchi E (2016): Aortic valve replacement: treatment by sternotomy versus minimally invasive approach. Braz J Cardiovasc Surg., 31:422-442.

9. Raja SG, Benedetto U, Amrani M (2013): Aortic valve replacement through $\mathrm{J}$-shaped partial upper sternotomy. J Thorac Dis., 5: 662-668.

10. Glauber M, Miceli A, Gilmanov D et al. (2013): Right anterior minithoracotomy versus conventional aortic valve replacement: a propensity score matched study. J Thorac Cardiovasc Surg., 145(5): 1222-6.

11. Mikus E, Calvi S, Tripodi A et al. (2013): Upper ' J' ministernotomy versus full sternotomy: an easier approach for aortic valve reoperation. J Heart Valve Dis., 22:295-300.

12. Reser D, Holubec T, Scherman J et al. (2015). Upper ministernotomy. Multimed Man Cardiothorac Surg., 2:2015. https://europepmc.org/article/med/26530961

13. Fudulu D, Lewis H, Benedetto $U$ et al. (2017). Minimally invasive aortic valve replacement in high risk patient groups. J Thorac Dis., 9:1672-1696. 